Quaderni di Geografia Cahiers de Gécongaty rataphie

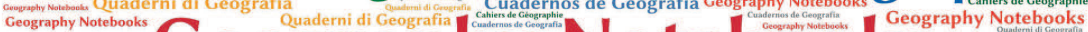
Cuadernos de Geografia Geography Notebooks $U 201000$ Cahiers de Géographi Cahiers de Géographie cahiers de Géographie Cuadernos de Geografía Cahiers de Géographie Cahiers de Géographie Cuadernos de Geografía Geography Notebooks

\author{
$4(2021)$ \\ 1 \\ Teatro di suoni. \\ Spazi acustici teatrali e territoriali
}

A cura di

Martino Mocchi, Lorena Rocca, Demis Quadri and Carlotta Sillano

EDITORIAL

Teatro di suoni per l'attaccamento ai luoghi. Uno sguardo geografico 11

Lorena Rocca

Per un teatro di suoni. Riflessioni su possibili dimensioni sonore nelle 23 creazioni site-specific di physical theatre

Demis Quadri

INTRODUCTION

Teatro di suoni. Spazi acustici teatrali e territoriali

Demis Quadri e Lorena Rocca

SPECIAL Issue

I suoni di Mantova come strumenti di interpretazione del paesaggio.

Tra turismo sostenibile ed educazione al patrimonio culturale

Valeria Pecorelli, Franca Zuccoli, Alessandra De Nicola, Enrico Squarcina

Il paesaggio sonoro campano tra contemporaneità e nuove forme

di progettualità turistica

Germana Citarella 
La narrazione spettacolarizzata del paesaggio sonoro.

Da Giuseppe Chiari a Philip K. Dick e oltre

Francesco Michi

Musica di paesaggi sonori. Enunciazione, risignificazione, comunicazione

Carlotta Sillano

Camminare per ascoltare. Partiture invisibili del territorio abitato

Elisabetta Senesi

Il paesaggio sonoro in relazione. Suono, movimento e immagini per stimolare complessità percettiva Angela Calia

Groove Fields. Understanding the Dance Floor from an Art-Based Research Perspective

Sebastian Mattbias

Il silenzio come esperienza trasformativa. L'importanza del silenzio nella meditazione e in ambito professionale

Sebastiano Caroni

Progettare il silenzio. Una lettura acustica dell'ex villaggio sanatoriale 125 Morelli a Sondalo

Martino Mocchi

Voicing One's Will. Theatre as Audio-Visual Hypotyposis of the Poetic

Michael Groneberg

Music and Clowning in Europe, 20th-21st centuries

Anna Stoll Knecht

Il paesaggio sonoro nella composizione musicale. Un percorso bibliografico

Stefano Alessandretti

\#exploreART: il labirinto di A. Pomodoro e i bambini. Un progetto di fruizione condivisa con percorsi sensoriali partecipati Alessandra De Nicola, Franca Zuccoli 


\section{OTHER EXPLORATIONS}

Il rumore lontano. Intervista a Lorena Rocca

a cura di Martino Mocchi

Re Cervo. Intervista a Antonella Astolfi

a cura di Krizia Bonaudo e Demis Quadri

Centovalli-Centoricordi. Intervista a Oliviero Giovannoni a cura di Krizia Bonaudo e Demis Quadri

Alla ricerca di un metodo: Open Space Technology 



\title{
Il silenzio come esperienza trasformativa. L'importanza del silenzio nella meditazione e in ambito professionale
}

\section{Sebastiano Caroni}

Docente di antropologia presso la Scuola superiore specializzata in cure infermieristiche (SSSCI), Bellinzona e Lugano

DOI: https://doi.org/10.7358/gn-2021-001-caro

\begin{abstract}
The article is motivated by the lack of studies which address silence as a context-dependent phenomenon; that is, as a phenomenon whose meaning arises within specific contexts, situations and physical spaces. Accordingly, I address the ways in which the phenomenon of silence plays an essential role in particular professional and vocational domains. The analysis leads through some thoughts and considerations of people who work or are active in specialized fields that have a significant connection with the practice and the experience of silence. I especially examine areas such as meditation, teaching, health professions, and other professional and even - to some extent - artistic practices.
\end{abstract}

Keywords: silence; professional field; meditation; teaching; psychotherapy.

Parole chiave: silenzio; ambito professionale; meditazione; insegnamento; psicoterapia.

\section{Il SILENZIO: UN FENOMENO CONTEXT-DEPENDENT}

Il silenzio assume significati diversi a partire dai contesti e dalle situazioni che ne informano il contenuto e il senso. Può essere vissuto come liberazione e fonte di autorealizzazione quando è praticato in piena libertà, ma può anche trasformarsi in strumento di potere e causare sentimenti 
di angoscia e oppressione; oppure, può suscitare imbarazzo quando è percepito come fuori luogo. Ma se il silenzio può essere tanto accogliente e aperto quanto freddo e chiuso ${ }^{1}$, come facciamo a riconoscerlo e a capirlo, valorizzandolo quando diventa accoglienza e liberandocene quando diventa chiusura? Il silenzio ci pone in contatto con la profondità del nostro essere, con le nostre esigenze e le nostre richieste più recondite. Pertanto, è importante conoscerne il valore, capire che è legato a dei contesti, a delle pratiche, a delle tecniche. Alcune persone ne sono particolarmente consapevoli, tanto che utilizzano il silenzio come risorsa nella loro professione, o più in generale nel loro ambito di specializzazione. $\mathrm{Ma}$ allora chi sono i professionisti del silenzio e dove si trovano? E come ci aiutano a ricostruire ciò che potremmo chiamare l'arte del buon uso, della conoscenza, e del rispetto del silenzio?

Questo contributo affronta il silenzio come fenomeno context-dependent, inserendolo cioè in una logica attenta al nesso fondamentale e alla compenetrazione fra il silenzio e i contesti in cui esso emerge e viene vissuto. La riflessione mi condurrà attraverso le testimonianze di persone attive in ambiti di specializzazione che intrattengono un nesso significativo con la pratica e l'esperienza del silenzio. Prenderò in esame soprattutto ambiti quali la meditazione, l'insegnamento, le professioni terapeutiche e sanitarie, e altri ambiti professionali o artistici in cui il silenzio riveste un ruolo centrale.

\section{UN'INDAGINE SUL SILENZIO IN AMBITO EDUCATIVO E NELLA PRATI- CA DELLA MEDITAZIONE}

In occasione di un corso di meditazione proposto a degli insegnanti di scuola media, e tenutosi tra febbraio e marzo del 2015 presso la Scuola media di Bellinzona, ho raccolto delle testimonianze scritte sul modo in cui gli insegnanti sperimentano il silenzio, sia nella loro abituale situazione d'insegnamento, sia nel contesto del corso di meditazione a cui prendevano parte ${ }^{2}$.

${ }^{1}$ Francesca Rigotti (2013) intraprende un'analisi delle metafore del silenzio evidenziando l'esistenza di silenzi freddi e solidi e, all'opposto, di silenzi più caldi e liquidi.

${ }^{2}$ Il corso era improntato agli insegnamenti del buddhismo Shambhala ed è stato condotto dal Prof. Daniele Bollini, insegnante di italiano e storia nel settore medio ticinese, dove opera dal 1990. Dal 1989 è istruttore e insegnante nella tradizione del buddismo Shambhala. Dal 1990 al 2005 è stato direttore del Centro Shambhala Ticino e

Geography Notebooks - 4 (2021) 1

https://www.ledonline.it/Geography-Notebooks/ 
Gli insegnanti che hanno preso parte all'esperienza, per molti insolita, hanno dapprima riflettuto sul ruolo del silenzio nel loro abituale contesto lavorativo. In questo senso, hanno riconosciuto unanimemente l'importanza del silenzio in contesti didattici, adducendo ragioni di diverso tipo, e insistendo sulla sua valenza pragmatica. Il silenzio è associato a momenti della lezione, a stati d'animo particolari oppure è ricercato come condizione che facilita l'apprendimento. Dalle testimonianze raccolte emerge che sovente gli insegnanti affermano di riuscire a ottenere il silenzio, ma ammettono che mantenerlo risulta problematico. In generale, gli insegnanti riportano esperienze, rappresentazioni e attitudini diverse riguardo alla pratica e all'osservazione del silenzio a scuola. Alcuni attingono agli strumenti gestionali che contraddistinguono il loro babitus professionale ricavandone e plasmando quelle strategie "vincenti" grazie alle quali ottengono il silenzio in classe. Ma quando ciò non avviene, è facile che subentri il disagio, perché l'assenza di silenzio può dare luogo a una serie di scenari negativi dove la turbolenza degli allievi spesso sfocia in comportamenti provocatori e offensivi.

\section{Dallinsegnamento alla meditazione}

Dopo aver raccolto le considerazioni dei docenti sul ruolo del silenzio nel loro contesto lavorativo, ho raccolto le loro impressioni riguardo al corso di meditazione nel quale erano stati coinvolti. In generale, il corso di meditazione è stato apprezzato dai docenti perché visto come un'occasione per conoscere meglio la meditazione sperimentandola in prima persona. Complessivamente, i momenti di meditazione sono stati vissuti sotto il segno della positività, del benessere e della calma. C'è stato chi non si è limitato a mettere in risalto la calma e la positività, ma ha ammesso che i momenti di meditazione hanno portato a galla anche molta agitazione. $\mathrm{E}$ c'è chi, ancora, ha fatto notare come durante una seduta di meditazione il benessere e l'armonia potevano lasciare spazio anche ad altre sensazioni: a volte si prova "benessere, calma e il piacere di meditare in gruppo, altre volte disagio, caos interiore e antipatia verso alcune persone".

Nel complesso, chi ha frequentato il corso di meditazione è stato positivamente marcato da un'esperienza in cui il silenzio ha giocato un ruolo fondamentale, nelle sue molte sfumature. Per un insegnante di

nel 2011 è stato nominato Shastri (esperto in testi e commentari) da Sakyong Mipham.

Geography Notebooks - 4 (2021) 1

https://www.ledonline.it/Geography-Notebooks/ 
educazione fisica il silenzio è "prevalentemente assenza di rumori a livello fisico e mentale (riuscire ad estraniarsi!)"; per altri invece assume una tinta decisamente più intimistica e spirituale ("per me significa: trovare me stessa”), oppure costituisce un'apertura verso il possibile: allora il silenzio diventa "il punto di partenza di molte cose: della comprensione di sé stessi e degli altri, dell'apprezzamento della realtà che ci sta attorno".

Se le esperienze di insegnamento e i primi assaggi di meditazione possono spesso apparire come due poli opposti, dovrebbe risultare altresì chiaro come tanto in un caso come nell'altro il silenzio costituisce una risorsa strategica determinante. Il contrasto, semmai, è riconducibile al carattere context-dependent del fenomeno e dell'esperienza. Il valore della risorsa silenzio viene quindi gestito, sperimentato, negoziato, eventualmente richiesto, mantenuto o ristabilito a seconda del contesto in cui ci si trova. Alcuni contesti, indubbiamente, sono più predisposti alla pratica e all'osservazione del silenzio. In attività e pratiche quali la meditazione, la lettura, la musica, e l'arte in generale, il silenzio può diventare un'esperienza più duratura, e proprio per questo più accessibile. Al tempo stesso, quale risorsa strategica, il silenzio può assumere un significato preciso proprio nella misura in cui ci si trova in un contesto determinato. Ma non solo: all'interno di uno stesso ambito professionale o specialistico, il valore del silenzio può essere rivendicato da alcuni ma sottovalutato o trascurato da altri.

\section{LA CURA DEL SILENZIO}

La storia della psicologia e delle tecniche psicoterapeutiche, per esempio, è caratterizzata, soprattutto negli ultimi decenni, da un fiorire di scuole, di approcci, tecniche, stili terapeutici, che si differenziano in modo piuttosto marcato gli uni dagli altri. Fra questi orientamenti, alcuni attribuiscono al silenzio un ruolo centrale nel percorso di cura, ma non tutti. Graziano Martignoni, psichiatra e psicoterapeuta, afferma quanto segue:

L'arcipelago psicoterapeutico è oggi più che mai variegato e spesso dissonante. Ognuno di noi, attori della Cura, farà discendere la sua scelta di metodo e la sua appartenenza teorica dalla propria visione del mondo e dalla propria idea di uomo. Vi sono, per dirlo con immagini certamente riduttive, i "palombari" o gli "astronauti", che amano la verticalità, che scelgono di essere esploratori del sottosuolo o dei cieli e vi sono sull'al- 
tro versante i "viaggiatori" e a volte persino i "turisti" della cura, che si accontentano della superfice su cui scivolare e sui cui almeno imparare a pattinare. Da questi orizzonti diversi discendono metodi e posture di cura, che hanno cura, valorizzano, esercitano e praticano il silenzio per le sue caratteristiche euristiche e terapeutiche, come avviene nella tradizione psicoanalitica, e altri al contrario che vivranno il silenzio come un tempo morto e sostanzialmente inutile o solamente sintomo di malattia. Io cerco di stare tra i palombari, non scordando la necessità sempre e comunque di essere compagno di strada di chi mi chiede aiuto. (Caroni 2016a)

All'interno dell'“arcipelago psicoterapeutico" il silenzio non è rivendicato unanimemente come risorsa curativa, tanto che se i "palombari" e gli “astronauti” - come li chiama Martignoni - «hanno cura, valorizzano, esercitano e praticano il silenzio per le sue caratteristiche euristiche e terapeutiche», i "viaggiatori" e i "turisti" della cura lo vivono «come un tempo morto e sostanzialmente inutile o solamente sintomo di malattia» (Ibid). Il valore attribuito al silenzio è quindi condizionato, in un campo come quelle della psicoterapia, tanto dalla scelta del metodo e dell'orientamento curativo, quanto da un'adesione personale, da parte del terapeuta, a una concezione del silenzio come tempo curativo non meramente passivo. Ecco cosa dice Martignoni:

Mi considero infatti, con tutta la modestia possibile, esploratore dei mondi interni dell'uomo, "viaggiatore in una stanza", come ci ricorda il poeta portoghese Fernando Pessoa. Per questo sento mio il pensiero di Jean Guitton, che parlando del silenzio claustrale scrive: "nei monasteri ciò che sorprende il visitatore di un giorno è la densità del silenzio, che, come si dice, vi regna. Il silenzio consiglia un rispetto sacro per l'interiorità dell'altro, una sola parola la violerebbe. Il silenzio non è presente in questi chiostri come un ospite o come un servitore: egli domina, regna nella pace e nella pienezza come un sovrano. Il silenzio ci introduce direttamente nel centro dell'altro, mentre la parola ci costringe a compiere lunghi e sinuosi giri periferici. Ecco perché nei monasteri votati al silenzio profondo, si parli sempre, ma non si chiacchieri mai”. (Ibid.)

La pregnanza del silenzio che caratterizza le pratiche monastiche viene qui ricondotta, traslata, e ricontestualizzata nell'ambito della pratica psicoterapeutica. L'analisi del silenzio come fenomeno e esperienza context-dependent non esclude, quindi, la contiguità, l'analogia e l'affinità fra 
esperienza del silenzio riconducibili a ambiti diversi.

Rimanendo nell'ambito della cura, vorrei insistere sull'importanza che i valori, l'impostazione, la sensibilità e le scelte del singolo operatore possono rivelare quanto alla possibilità che il silenzio si riveli come risorsa strategica all'interno di un ambito o di un percorso professionale. Nicole De Lorenzi, per esempio, da quindici anni si occupa di accompagnamento alla morte presso l'ospedale di Winterthur - un lavoro che consiste nel sostenere e accompagnare la persona che sta morendo e, a seconda dei casi, anche i famigliari - riconoscendo al silenzio un potenziale terapeutico. Come rivela in un'intervista, il suo indirizzo professionale è intrecciato alla sua biografia:

Quando avevo quattordici anni mia mamma ha subito un'emorragia cerebrale ed è rimasta per tredici mesi in coma. Dopo tredici mesi è morta. È stato un periodo molto intenso per me, così come per mia sorella e mio padre. Un carosello di speranza e disperazione. Questa esperienza mi ha scosso nel profondo, mi sono anche molto arrabbiata. Poi, crescendo, ho intrapreso un cammino spirituale e ho studiato teologia, nella speranza di trovare risposte a domande come: perché viviamo? Che senso ha? Grazie allo studio sono riuscita a formulare meglio le mie domande, e per fortuna ho ricevuto anche qualche chiara risposta. (Caroni 2016b, 54)

Un percorso biografico lungo il quale, anche grazie alla preghiera contemplativa, ha gradualmente scoperto il valore del silenzio nella relazione di cura:

Noto che le persone vicine alla morte diventano sempre più essenziali e hanno sempre meno bisogno di interazione o dialogo. Cercano la calma, la pace. Non vogliono essere disturbate, non hanno energia per interagire. Però spesso sanno approfittare di una persona capace di stare in silenzio. Il silenzio non è solo tacere. È più profondo. Ė l'essere nel momento, né nel passato, né nel futuro. Presenti nel presente. È una qualità che viene percepita e apprezzata da persone che non hanno più le forze per interagire. (Ibid.)

Se la relazione umana e terapeutica invita a riconoscere il silenzio quale elemento significativo, d'altra parte è l'operatore o operatrice che, con la collaborazione del paziente, lo interpreta e ne attiva le potenzialità. 


\section{SILENZI BIOGRAFICI}

Quanto discusso finora mostra come la trasformazione del silenzio in risorsa professionale viene realizzata quando si prefigura uno spazio di convergenza fra la predisposizione del singolo (o del gruppo) e le caratteristiche specifiche del contesto professionale o ambito di specializzazione in cui si opera. Come racconta l'ornitologa e musicista Anita Python, uno specifico ambiente lavorativo può predisporre all'esperienza del silenzio:

Quando sono sul terreno il silenzio è molto importante per permettermi di rilevare la presenza degli uccelli, soprattutto quando sono nascosti nella corona degli alberi. È spesso solo grazie ai loro canti e richiami che riesco a identificarli e a censirli. Gli uccelli stessi sono influenzati dai rumori circostanti. In città i merli e i codirossi sono i primi a svegliarsi e a riempire il silenzio della notte con il loro canto. Quando poi il rumore del traffico si fa sentire, loro tacciono. Il loro comportamento territoriale, infatti, si basa principalmente sulla comunicazione uditiva. Per i rapaci notturni il silenzio addirittura è indispensabile per poter localizzare le loro prede. Ė grazie all'udito che riescono a scovare piccoli mammiferi anche nell'erba o sotto la neve. In un certo senso anche io, come biologa, vado a caccia di suoni che segnalano la presenza di animali [...]. Quando mi trovo sul campo, è più facile per me basarmi sull'udito piuttosto che sulla vista. Questo implica che non ci siano altri rumori di fondo. (Caroni 2016c)

Anche per Anita, così come per Nicole De Lorenzi, la formazione professionale si intreccia con la biografia. Anita ha iniziato lo studio del violino all'età di 6 anni, e da più di 10 anni si dedica anche alla viola. Quando si tratta di musica, per lei «il silenzio è la base, sia durante lo studio, dove l'ascolto e l'attenzione al proprio strumento sono indispensabili, sia durante le esecuzioni» (Ibid). E non è un caso se la sua passione per la musica le permette di affinare la sensibilità sul lavoro, in particolare quando si tratta di riconoscere le specie di uccelli dai loro canti: «credo in particolare che l'esperienza acquisita con la musica mi abbia molto aiutato nell'apprendimento dei canti. La capacità di visualizzare gli intervalli tra i suoni, quantificare la loro lunghezza, definire il ritmo, individuare la presenza di strofe è molto utile» (Ibid).

A supporto della valorizzazione del silenzio in un contesto professionale troviamo spesso un percorso biografico, che aiuta a capire come il silenzio possa attivarsi come risorsa sul posto di lavoro. Ma, d'altra 
parte, si può anche rovesciare la prospettiva per cogliere come ogni silenzio sia già, essenzialmente, biografico. Il regista e documentarista Olmo Cerri, in occasione della rassegna culturale "Sensi del silenzio" tenutasi a Bellinzona nel 2014, ha allestito un'opera video che mette in scena le variazioni espressive generate dallo stare in silenzio. Chiedendo a delle persone di rimanere sedute in silenzio davanti a una telecamera, è giunto alla seguente conclusione:

Il silenzio è personale e può essere quindi biografico. Ognuno di noi ha un modo diverso di "stare in silenzio" e di gestirlo, confrontandosi con i fantasmi che porta con sé: l'imbarazzo (per la richiesta particolare e per la presenza di una telecamera), la gestione del tempo (un minuto stimato è molto diverso per ognuno), l'impossibilità di rimanere in silenzio per cause esterne (notifiche sul cellulare, colpi di tosse, bisogno di commentare o di parlare fra sé e sé). Restando in silenzio sveliamo particolari di noi che spesso sono coperti e mascherati dalla nuvola di parole e rumori che produciamo. (Caroni 2016d)

Seduti, in silenzio, continuiamo a comunicare agli altri chi siamo.

\section{IL SILENZIO COME ESPERIENZA TRASFORMATIVA}

Il silenzio, come abbiamo messo in evidenza nelle fasi introduttive di questo documento, può essere associato a momenti e sensazioni tanto positive quanto negative. Data la complessità e la natura ambivalente del fenomeno, mostrare il suo carattere context-dependent ci aiuta a capire come all'interno di situazioni professionali o aree di specializzazione, il contesto interviene come elemento di mediazione, come spazio più o e meno strutturato, che favorisce, informa e incoraggia l'esercizio, la pratica, e l'osservazione del silenzio. D'altra parte abbiamo pure messo in evidenza quanto la predisposizione del singolo sia importante affinché il silenzio si trasformi in una risorsa al servizio del singolo e del gruppo. Il silenzio gioca spesso un ruolo importante nel percorso biografico di coloro che ne riconoscono il pieno valore. Riteniamo quindi che sia importante riconoscere che l'uso del silenzio quale risorsa specifica sia da ricondurre a una felice convergenza fra le caratteristiche di un ambito professionale (tenendo conto del significato più vasto del termine professione) e le predisposizioni individuali, spesso legate a un percorso biografico particolare. Quando questa convergenza si realizza, allora il silenzio 
diventa un'esperienza che facilmente travalica i confini di ambiti specifici socialmente definiti e circoscritti, per diventare autentico stile di vita.

D'altra parte, sulla scorta di queste osservazioni, possiamo anche chiederci ciò che accomuna le diverse esperienze di silenzio su cui ci siamo soffermati in questo scritto. Si potrebbe ipotizzare, per esempio, che le esperienze di silenzio che abbiamo riportato siano trasformative, nel senso che incidono direttamente sull'interiorità del soggetto. Christopher Bollas (2018) definisce il concetto di oggetto trasformativo in riferimento al rapporto fra il neonato e la madre. Il rapporto fra il bambino e la madre si configura come rapporto produttivo di sensazioni grazie a cui il neonato diventa progressivamente soggetto indipendente e si individualizza. Tracce di questo rapporto primordiale permangono anche negli adulti, e spesso il rapporto fra lo psicanalista e il paziente ripropone questo rapporto privilegiato. Nella relazione terapeutica, inoltre, il paziente può sperimentare un tipo di silenzio grazie a cui può riavvicinarsi e rivivere momenti appartenenti a esperienze passate lasciato nell'ombra. Nel contesto analitico descritto da Bollas, il silenzio può diventare un'esperienza che trasforma l'interiorità, sia perché permette il recupero di stati emotivi passati, sia perché genera nuove opportunità di relazionarsi al presente ${ }^{3}$. Un'ipotesi interessante, che speriamo di poter approfondire in un prossimo contributo ${ }^{4}$.

\section{RIFERIMENTI BIBLIOGRAFICI}

Bollas, C. 2018. The Shadow of the Object [ed. or. 1987]. London: Routledge.

Caroni, S. 2016a. "Un silenzio terapeutico. Intervista a Graziano Martignoni”. laRegioneTicino. 11 giugno 2016.

Caroni, S. 2016b. "Sulla soglia, in silenzio. Intervista a Nicole De Lorenzi". Rivista per le Medical Humanities 34 (maggio-agosto 2016): 54.

Caroni, S. 2016c. "Su un manto di silenzio. Fra musica e natura, conversazione con Anita Python”. laRegioneTicino. 2 aprile 2016.

${ }^{3} \mathrm{Si}$ veda in particolare il capitolo 14 , "Ordinary regression to dependence" (Bollas 2018, 173-185).

${ }^{4}$ In particolare, sarebbe interessante indagare il nesso che intercorre fra il silenzio interiore e l'esperienza dell'io inteso come progetto autoriflessivo. Tanto il silenzio interiore quanto l'autoriflessività sono, infatti, intimamente legati al progetto della modernità (Giddens 1991). 
Caroni, S. 2016d. "Seduti il silenzio. I molti significati dello stare zitti, conversazione con Olmo Cerri”. laRegioneTicino. 7 maggio 2016.

Comolli, G. 2012. Una luminosa quiete. La ricerca del silenzio nelle pratiche della meditazione Milano: Mimesis.

Corbin, A. 2016. Une histoire du silence: de la Renaissance à nos jours. Paris: Albin Michel Gasparini, G. 2012. C’è silenzio e silenzio. Forme e significati del tacere. Milano: Mimesis.

Giddens, A. 1991. Modernity and Self-Identity. Cambridge: Polity Press.

Le Breton, D. 1997. Du silence. Paris: Métaillé.

Lees, H. E. 2012. Silence in Schools. Stoke-on-Trent Trentham Books.

Rigotti, F. 2013. Metafore del silenzio. Milano: Mimesis. 